Article

\title{
Error Averaging Effect in Parallel Mechanism Coordinate Measuring Machine
}

\author{
Peng-Hao Hu *, Chang-Wei Yu, Kuang-Chao Fan, Xue-Ming Dang and Rui-Jun Li \\ School of Instrument Science and Opto-electronic Engineering, Hefei University of Technology, \\ Hefei 230009, China; hfutycw@hfut.edu.cn (C.-W.Y.); fan@ntu.edu.tw (K.-C.F.); \\ dangxm@hfut.edu.cn (X.-M.D.); rj-li@hfut.edu.cn (R.-J.L.) \\ * Correspondence: hupenghao@hfut.edu.cn; Tel./Fax: +86-551-62902797
}

Academic Editor: Richard Leach

Received: 15 September 2016; Accepted: 18 November 2016; Published: 25 November 2016

\begin{abstract}
Error averaging effect is one of the advantages of a parallel mechanism when individual errors are relatively large. However, further investigation is necessary to clarify the evidence with mathematical analysis and experiment. In the developed parallel coordinate measuring machine (PCMM), which is based on three pairs of prismatic-universal-universal joints (3-PUU), error averaging mechanism was investigated and is analyzed in this report. Firstly, the error transfer coefficients of various errors in the PCMM were studied based on the established error transfer model. It can be shown how the various original errors in the parallel mechanism are averaged and reduced. Secondly, experimental measurements were carried out, including angular errors and straightness errors of three moving sliders. Lastly, solving the inverse kinematics by numerical method of iteration, it can be seen that the final measuring errors of the moving platform of PCMM can be reduced by the error averaging effect in comparison with the attributed geometric errors of three moving slides. This study reveals the significance of the error averaging effect for a PCMM.
\end{abstract}

Keywords: error averaging effect; parallel mechanism; parameter error; coordinate measuring machine

\section{Introduction}

Error averaging effect has always existed in precision measurement, which is an important factor in improving accuracy. Sensors such as capacitance sensor, linear grating, and inductosyn have averaging effects in reading data. For instance, if a rotary encoder is installed with two reading heads with a $180^{\circ}$ separation, the eccentricity error of the grating disk with respect to the shaft can be eliminated [1]. Another example can be seen on an end-tooth precision indexing table: several teeth of the upper gear and the lower gear are engaged at the same time during lapping or indexing, and individual tooth errors are averaged and reduced [2]. Hydrostatic bearing or air-bearing spindles and stages also perform with high accuracy using error averaging effect [3]. Considerable researches on the error averaging effect in precision engineering have been conducted to reduce overall errors in the past. In the serial-type machine or machine tool, the volumetric errors are cumulated from individual geometric errors. Although, the parallel-type mechanism has the error averaging effect, it has been rarely studied [4].

Generally speaking, the error sources in the parallel mechanism can be classified into two categories: one error source always exists in common precision machines due to assembly and applied load, such as geometric errors in each axis, spindle motion error, and deformation error on account of heat and force; the other error originates from the parallel mechanism itself, such as input motion error, structure parameter error, component assembled error, location error of parts, and clearance error of joints. All these errors contribute to the positional error of the end functional point of the moving platform. When the parallel mechanism works, however, all these errors will have influence on one 
another when transferring to the moving platform (i.e., the error averaging effect). In recent years, we focused on the development of a three-pair prismatic-universal-universal joint (3-PUU) parallel coordinate measuring machine (PCMM). Some phenomena about error averaging effect have been observed due to parameter errors. This study aims to reveal the essence of error averaging effect due to geometric errors.

\section{Measuring Principle of 3-PUU PCMM}

The structure of the developed PCMM is shown in Figure 1. Three sliders are connected to the moving platform through universal joints and three pairs of link rods. The sliders share one horizontal linear guider and can move separately while driven by steel belts and stepper motors, respectively. The linear grating ruler is fastened to one side of the linear guide. Three reading heads are installed with respect to each slider. The moving platform is connected to the end of each link rod through universal joints, and the moving platform translates in the space parallel to the three sliders without rotation. The trigger probe assembled on the moving platform triggers three reading heads to collect the instantaneous positions synchronously, when the probe touches workpiece $[5,6]$. The coordinate of the probe can then be calculated based on a measuring model $[7,8]$.

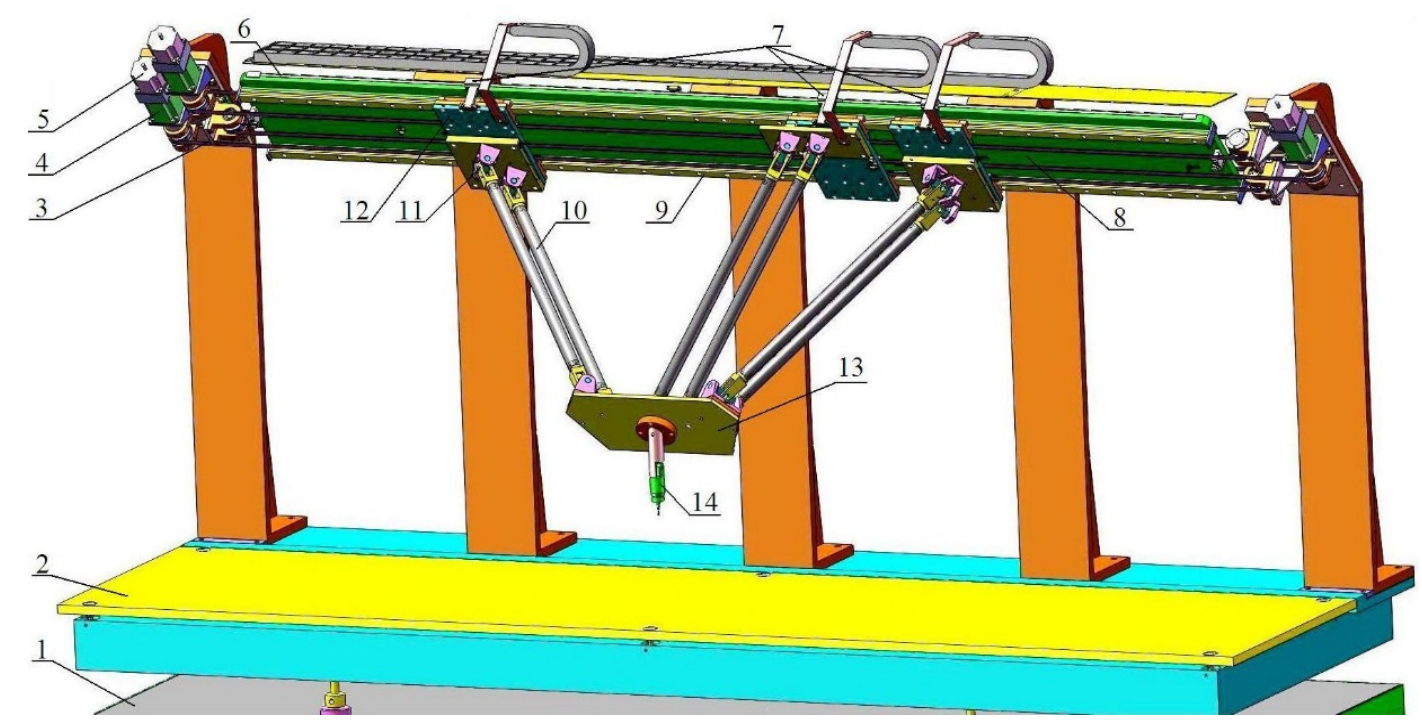

(a)

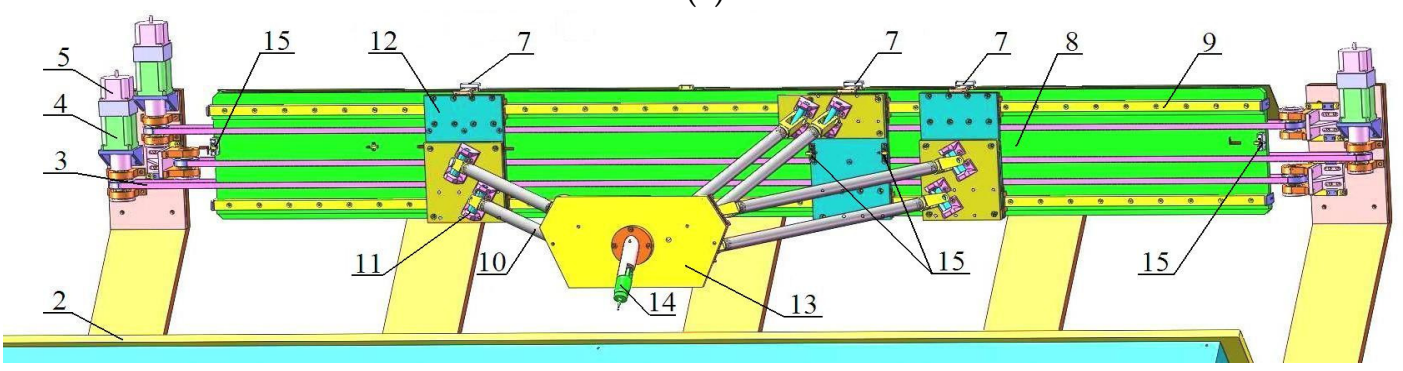

(b)

Figure 1. 3-PPU (three pairs of prismatic-universal-universal joints) PCMM (parallel coordinate measuring machine). (1) Base, (2) table, (3) steel belt, (4) reducer, (5) motor, (6) linear grating, (7) reading head, (8) beam, (9) linear guider, (10) rod, (11) universal joints, (12) slider, (13) moving platform, (14) measuring head, and (15) limit switch. (a) front view; (b) upward view.

As shown in Figure 1, three sliders are coplanar, thus, the centers of six universal joints on three sliders also share one common plane, which is plane $O C D B$ in Figure 2. The angle between the $O C D B$ plane and the horizontal plane of $O H P B$ is $45^{\circ} . B_{1}, B_{2}$ are centers of two joints on the left slider; $B_{3}$, 
$B_{4}$ are centers of the middle slider; and $B_{5}$ and $B_{6}$ are centers of the right slider. A fixed coordinate system $O-X Y Z$ is established based on the plane $O C D B$, and every center of universal joints $B_{i}$ can be expressed as: $B_{1}\left(x_{1}, 0,0\right), B_{2}\left(x_{1}, c, 0\right), B_{3}\left(x_{2}, b+c, 0\right), B_{4}\left(x_{2}+a, b+c, 0\right), B_{5}\left(x_{3}, c, 0\right), B_{6}\left(x_{3}, 0,0\right)$. Here, $x_{1}$, $x_{2}, x_{3}$ indicate the positions of three reading heads, respectively, and $a, b, c$ are position parameters between joints on three sliders. The moving platform is in the form of an uneven six-sided polygon on which $e_{i}$ is the center of universal joint $i$ on the moving platform. Let point $e_{1}$ be the unknown position in the coordinate system of $O-X Y Z$ and set it to $(x, y, z)$. Thus, $e_{2}-e_{6}$ coordinates can be determined with position parameters $(e, f, g)$ between joints. According to the spatial two-point formula, a set of equations expressing all link lengths can be established as below [9].

$$
\left\{\begin{array}{l}
\left(x-x_{1}\right)^{2}+y^{2}+z^{2}=l_{1}^{2} \\
\left(x-x_{1}\right)^{2}+(y+g-c)^{2}+z^{2}=l_{2}^{2} \\
\left(x+e-x_{2}\right)^{2}+(y+g+f-b-c)^{2}+z^{2}=l_{3}{ }^{2} \\
\left(x+d+e-a-x_{2}\right)^{2}+(y+g+f-b-c)^{2}+z^{2}=l_{4}{ }^{2} \\
\left(x+d+2 e-x_{3}\right)^{2}+(y+g-c)^{2}+z^{2}=l_{5}^{2} \\
\left(x+d+2 e-x_{3}\right)^{2}+y^{2}+z^{2}=l_{6}^{2}
\end{array}\right.
$$

The group consists of six equations and only three unknowns. If two rods have the same length on each slider (i.e., $l_{1}=l_{2}, l_{3}=l_{4}, l_{5}=l_{6}$ ), the unknown position of point $e_{1}$ can be solved as:

$$
\left\{\begin{array}{l}
x=\frac{x_{1}+x_{3}-2 e-d}{2}+\frac{l_{5}^{2}-l_{1}^{2}}{2 e+d-x_{3}+x_{1}} \\
y=\frac{l_{3}^{2}-l_{1}^{2}+\left(x-x_{1}\right)^{2}-\left(x+e-x_{2}\right)^{2}}{2(f-b)}+\frac{b-f}{2} \\
z=\sqrt{l_{1}^{2}-\left(x-x_{1}\right)^{2}-y^{2}}
\end{array}\right.
$$

The probing position in space can be obtained from point $e_{1}$ with a transformation matrix containing sliders' positions $\left(x_{1}, x_{2}, x_{3}\right)$ and structural parameters of link $l_{i}(i=1-6)$, joints positions $(a, b, c, d, e, f, g)$, and the stylus length, if the probe-mounting position on the moving platform is known. In this study, we only focus on the motion errors of sliders that would affect the positional errors of the probe and, therefore, assume all structural parameters to be constant. In such a case, the probing position in space $\left(x_{p}, y_{p}, z_{p}\right)$ can be simplified to the function of sliders positions as below.

$$
\left\{\begin{array}{l}
x_{\mathrm{p}}=f_{1}\left(x_{1}, x_{2}, x_{3}\right) \\
y_{p}=f_{2}\left(x_{1}, x_{2}, x_{3}\right) \\
z_{p}=f_{3}\left(x_{1}, x_{2}, x_{3}\right)
\end{array}\right.
$$

Equations (2) and (3) are measuring models that are also called positive solution in parallel mechanism. The positive solution is the key in analyzing the working space of the probe and establishing the error measuring model. The working space refers to the set of points in space wherein the trigger probe can reach. The measuring accuracy is closely related to the position of the probe tip within the working space. For a specified measuring accuracy, however, only within a certain range of working space can the accuracy of the PCMM achieve the required level. This range is called the measurement space. The measurement space is expected to be as large as possible and is largely dependent upon the accuracy of each slider's motion and each structural parameter. Based on the required working space of parallel mechanism and the accuracy index as target, the optimization design with respect to all structural parameters of the PCMM was conducted $[10,11]$. In this study, the motion errors of each slider were measured, and the error averaging effect to the probing position is analyzed in the following sections. 


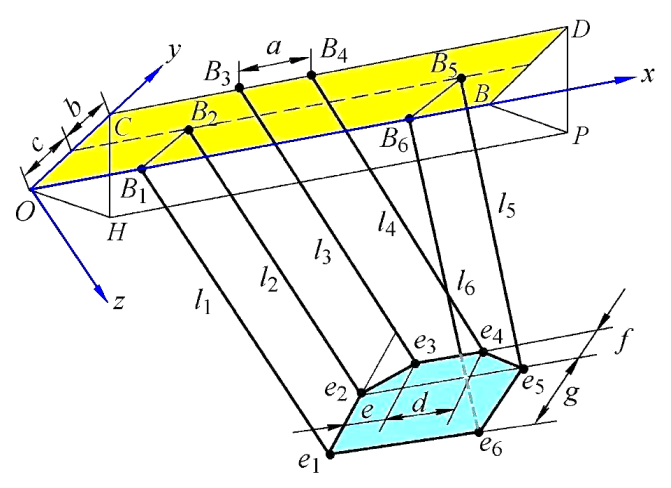

Figure 2. Geometrical model of PCMM.

\section{Analysis of Grating Reading Error}

It can be seen from Equation (3) that the measuring result depends on the positions of three reading heads. The reading error of the grating scale, which is often called "input motion error" in the parallel mechanism, affects the measuring error. The sensitivity of reading error to measuring error, called uncertainty analysis, can be derived from Equation (3) with differential coefficient theory.

$$
\left[\begin{array}{l}
\delta_{1} x \\
\delta_{1} y \\
\delta_{1} z
\end{array}\right]=\left[\begin{array}{lll}
\frac{\partial f_{1}}{\partial x_{1}} & \frac{\partial f_{1}}{\partial x_{2}} & \frac{\partial f_{1}}{\partial x_{3}} \\
\frac{\partial f_{2}}{\partial x_{1}} & \frac{\partial f_{2}}{\partial x_{2}} & \frac{\partial f_{2}}{\partial x_{3}} \\
\frac{\partial f_{3}}{\partial x_{1}} & \frac{\partial f_{3}}{\partial x_{2}} & \frac{\partial f_{3}}{\partial x_{3}}
\end{array}\right]\left[\begin{array}{l}
d x_{1} \\
d x_{2} \\
d x_{3}
\end{array}\right]
$$

where, $\left[\delta x_{p}, \delta y_{p}, \delta z_{p}\right]^{\mathrm{T}}$ are measuring errors while $\left[d x_{1}, d x_{2}, d x_{3}\right]^{\mathrm{T}}$ are reading errors. The middle $3 \times 3$ matrix is called the error transfer coefficient matrix.

Putting practical values of the PCMM structure parameters into Equations (2) and (3), we can derive the error transfer coefficient matrix of every point in the measuring space. By conducting substantial simulation calculation, we found that the PCMM averaged the original reading head errors through link rods. Table 1 lists the error transfer coefficients of two randomly selected points in the measuring space. Other points generally have the same characteristics.

Table 1. Error transfer coefficient of two points.

\begin{tabular}{cccc}
\hline $\begin{array}{c}\text { Serial } \\
\text { Number }\end{array}$ & $\left(\partial f_{i} / \partial x_{1}\right) / \mathbf{m m}$ & $\left(\partial f_{i} / \partial x_{2}\right) / \mathbf{m m}$ & $\left(\partial f_{i} / \partial x_{3}\right) / \mathbf{m m}$ \\
\hline \multicolumn{4}{c}{$(x, y, z)=(600,420,240)$} \\
$i=2$ & 0.5 & 0 & \\
$i=3$ & 0.13 & 0.16 & 0.5 \\
& -0.11 & -1.24 & 0.43 \\
\hline$i=1$ & $(x, y, z)=(600,360,290)$ & 0.5 \\
$i=2$ & 0.5 & 0 & 0.13 \\
$i=3$ & -1.13 & 1.26 & -0.23 \\
\hline
\end{tabular}

As given in Equation (2), the $x$ coordinate of point $e_{1}$ is determined only by the first and the third reading head data. The value of the second reading head is not related. The error transfer coefficient of the first and the third reading head are the same at 0.5 . The error transfer coefficient of the second reading head is zero. In other words, if the length of the first and the third link rod are equal (i.e., $\left.l_{1}=l_{2}=l_{5}=l_{6}\right)$, then $x$ coordinate of point $e_{1}$ will be equal to $\left(x_{1}+x_{3}-2 e-d\right) / 2$. This phenomenon is very close to what we conduct in angle measurement, with two reading heads installed $180^{\circ}$ apart on the circular grating. In the $Y$ and $Z$ directions, positive and negative error transfer coefficients 
alternately appear, and the sum tends to zero. Therefore, we can conclude that three grating reading head errors have been averaged in the PCMM. In other words, the error average effect of the parallel mechanism is embodied in the motion input errors.

Using the same total differential method, we have studied the length manufacturing error of six link rods and its influence on the measuring error. A similar conclusion was determined. For the $X$ direction, the error transfer coefficient could realize compensation by itself. In $Y$ and $Z$ direction, the error averaging effect still exists, but is weaker than that in the $X$ direction [11].

\section{Property of Slider Motion Errors in PCMM}

Linear motion stage is always employed in precision engineering. The stage provides straight line motion reference, which is expected to travel along a straight line. However, in practice, the actual path and orientation of the stage deviate from the straight line of motion because of its own geometric errors and assembling errors. As shown in Figure 3, five unexpected motions-two straightness motion errors and three angular motion errors-occur in five degrees of freedom (5-DOF). The motion straightness errors include two linear errors along $Y$ and $Z$ direction, respectively. The angular motion errors around each axis are called pitch, yaw, and roll.

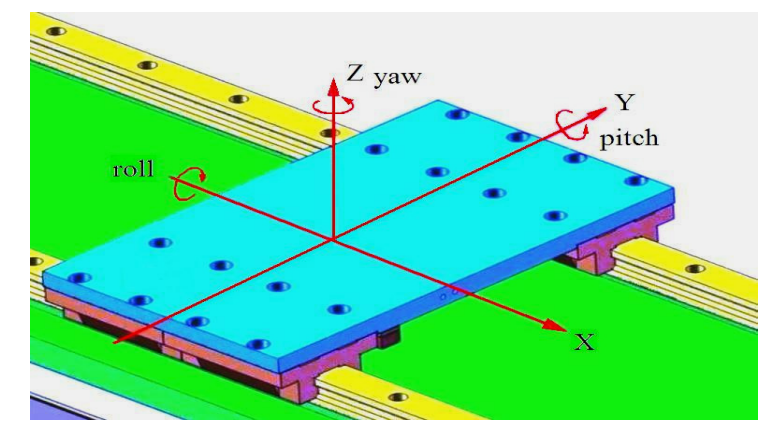

Figure 3. Degrees of freedom (DOFs) of the stage.

Over the years, numerous scholars have intensively studied the characteristics of the aforementioned geometric errors on precision machines and made many valuable contributions, especially in analysis and discussion of the relationship between the motion straightness errors and angular motion errors. Their conclusion can be summarized as follows [12-17]:

(1) The straightness motion error of the linear stage is closely related to the straightness error of the guide rail itself, but these errors are different in that the guide rail straightness error is a "cause", whereas the straightness motion error is a "result";

(2) The angular motion error and motion straightness error are also related, but no definite function relation exists between them;

(3) When a different point on the stage is measured, its value of straightness motion error may differ.

Our study showed that the motion straightness error of the slider is the key error source that influences and even determines the accuracy and performance of the PCMM. This is because since the three sliders are shared with only one linear guider rail, their motion errors will have an impact on the moving platform through six link rods. Figure 4 illustrates one slider working on the guide rail. A detailed discussion about the slider angular motion errors and motion straightness errors will be given, respectively, in the following sections. 


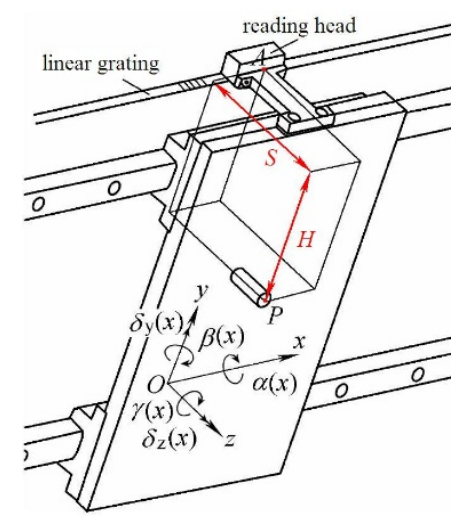

Figure 4. Slider motion in PCMM.

\subsection{Abbe Error and Angular Motion}

The Abbe principle, which was proposed in 1890, is often referred to as the first principle for instrument design. This principle could avoid or eliminate the first-order measuring error caused by angular motion error. The Abbe error also plays a key role in 3-PUU PCMM. As shown in Figure 4, point $\mathrm{P}$ is the center of one joint on the slider, while point $\mathrm{A}$ is the center of grating reading head. Two points are located at the different corners of the cuboids diagonal. Distance $H$ and $S$ are Abbe offsets, which are perpendicular to each other in $Y$ and $Z$ directions, respectively. Pitch $\beta(x)$ and yaw $\gamma(x)$ of the slider produce Abbe error of point $\mathrm{P}$ with respect to point $\mathrm{A}$. When the slider moves to a point $x_{i}$, its yaw angle is $\gamma\left(x_{i}\right)$. This means that the slider will rotate a small angle around the $Z$ axis, which changes the position of reading head to linear grating; this is one kind of Abbe error which is equal to Abbe offset $H$ times $\gamma\left(x_{i}\right)$. The pitch $\beta\left(x_{i}\right)$ also produces similar results with another Abbe offset $S$. Thus, total Abbe error can be expressed as Equation (5). This issue can be analyzed as an input error. The method is similar to the analysis of the sensor reading error in part 3 [18-20].

We substitute the grating reading error in Equation (4) with Abbe error. Here, $\mathrm{d} x_{i}$ can be expressed by:

$$
\mathrm{d} x_{i}=-H_{i} \tan \gamma\left(x_{i}\right)-S_{i} \tan \beta\left(x_{i}\right)
$$

As the pitch and yaw angle is very small, Equation (5) can be simplified as:

$$
\mathrm{d} x_{i}=-H_{i} \gamma\left(x_{i}\right)-S_{i} \beta\left(x_{i}\right)
$$

In an experiment, the pitch and yaw angular motion errors of three sliders (HIWIN Technologies Corp., Taichung, China) were measured by an autocollimator (Jingda Measurement Technology Co. Ltd., Jiujiang, China), as shown in Figure 5. The overall trends of the three sliders' angle errors were very similar. Figure 6 shows the angular motion data of the left slider, which contains 37 points. Angular data are collected once after the slider moves $50 \mathrm{~mm}$. Thus, the serial number of the horizontal axis in Figure 6 is corresponds to the different positions of the left slider. Several models of spline curve interpolation for the angular motion error of three sliders were established after obtaining experimental data, which was important for completing the subsequent error calculation.

As shown in Table 2, 3 cross-sections $(x=750,1000,1250)$ in the measurement space are selected initially, and 9 points are selected as feature points in each section. Therefore, 27 points are distributed in the measurement space. Corresponding to these points are each slider's position coordinate, which can be determined through the inverse kinematic solution of PCMM using Equation (2). Thus, the pitch and yaw angle error value of every slider can be achieved through the angle error model. Putting measured angle errors of Figure 6 into in Equations (3) and (6), we can determine the contribution of Abbe errors of the three sliders to the probe's positional errors in the measurement space as listed in Table 2. 


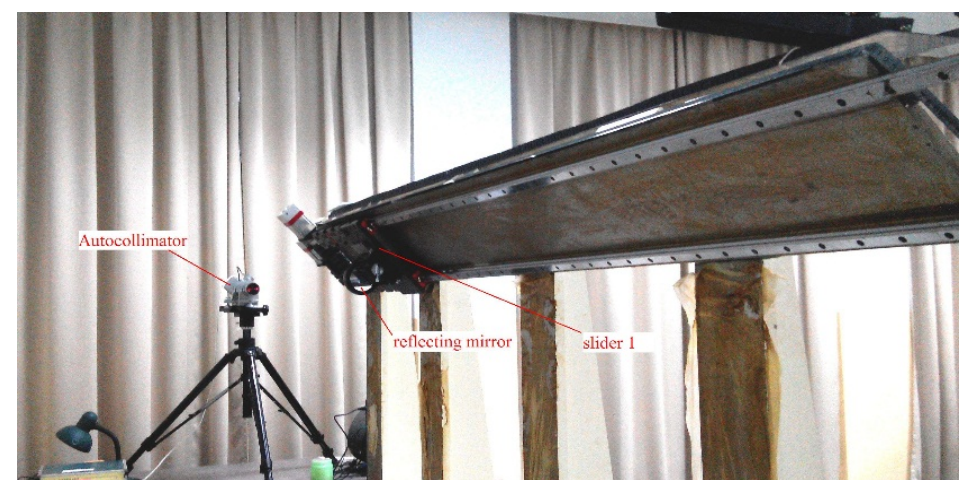

Figure 5. Autocollimator detection.
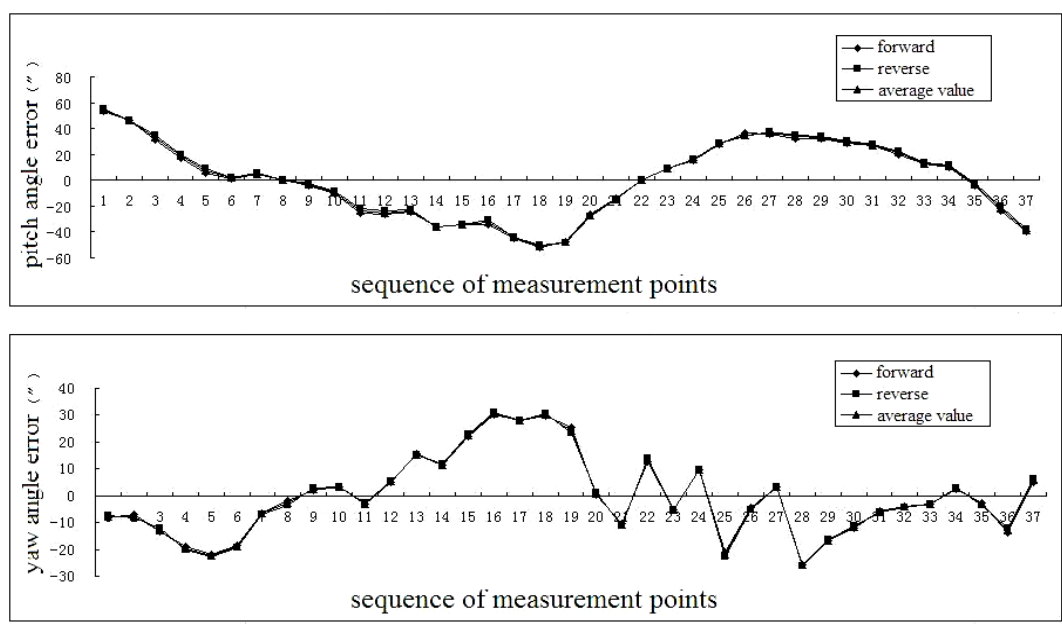

Figure 6. Pitch and yaw angle error of slider 1.

Table 2. Error contributed by Abbe error of three sliders.

\begin{tabular}{ccccccc}
\hline Selected Points/mm & \multicolumn{2}{c}{ Corresponding Slider Position/mm } & \multicolumn{3}{c}{ Error Value/ $\mathbf{m m}$} \\
\hline$(X, Y, Z)$ & $x_{1}$ & $x_{2}$ & $x_{3}$ & $\delta_{x A}$ & $\delta_{y A}$ & $\delta_{z A}$ \\
\hline $750,150,200$ & 193.58 & 1167.44 & 1319.58 & -7.60 & -21.96 & -12.13 \\
$750,150,300$ & 240.49 & 1047.23 & 1285.55 & 2.53 & 19.79 & 6.84 \\
$750,150,400$ & 314.56 & 1074.49 & 1211.45 & 4.59 & 16.38 & 11.68 \\
$750,300,200$ & 257.96 & 1357.98 & 1468.03 & 2.55 & -15.23 & 5.77 \\
$750,300,300$ & 311.70 & 1308.28 & 1414.32 & 5.90 & 17.06 & 15.68 \\
$750,300,400$ & 400.57 & 1228.27 & 1325.46 & -5.69 & -20.05 & 1.74 \\
$750,450,200$ & 390.01 & 1371.35 & 1486.67 & -2.79 & -18.34 & -1.57 \\
$750,450,300$ & 467.86 & 1310.48 & 1458.19 & 1.28 & 7.87 & 1.40 \\
$750,450,400$ & 652.02 & 1203.11 & 1374.04 & -5.36 & 14.45 & 1.97 \\
$1000,150,200$ & 443.58 & 1403.64 & 1582.45 & -3.57 & -14.40 & 4.15 \\
$1000,150,300$ & 490.49 & 1397.26 & 1735.50 & -3.52 & -11.86 & -0.25 \\
$\ldots$ & $\ldots$ & $\ldots$ & $\ldots$ & $\ldots$ & $\ldots$ & $\ldots$ \\
$1250,450,200$ & 890.00 & 1671.33 & 1836.56 & 3.11 & 17.34 & 4.51 \\
$1250,450,300$ & 967.86 & 1610.46 & 1858.18 & 2.62 & 17.07 & 3.33 \\
$1250,450,400$ & 1152.0 & 1653.10 & 1874.07 & 2.27 & -11.78 & -2.10 \\
\hline
\end{tabular}

... represents a few sets of data was omitted, because there are 27 sets of data in table, if all data are shown, the table will occupy too large columns.

Based on the calculation results, for 27 selected feature points, it can be seen that the contribution of the angular motion errors to measuring accuracy of the probe in the $X$ direction is the smallest. Error variation fluctuates from -7.6 to $+5.9 \mu \mathrm{m}$. The error contribution to the $Y$ direction is the largest, 
ranging from -21.9 to $\sim+22.3 \mu \mathrm{m}$. The error variation can be observed in Figure 7. Compared with the errors in $Y$ and $Z$ directions, the Abbe error has a minimal effect in the $X$ direction because the error averaging effect in $X$ direction is strongest. It is similar to the results of our previous reports on reading head error analysis [21].

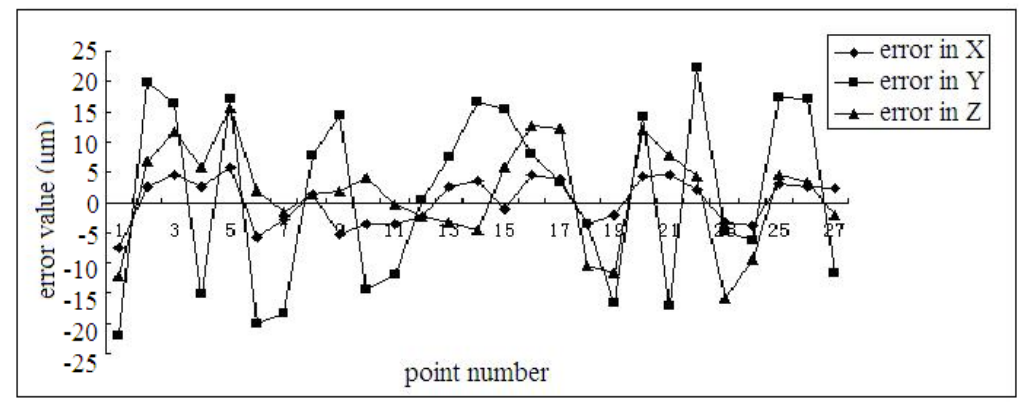

Figure 7. Variation of error caused by Abbe error.

\subsection{Straightness Motion Error}

As shown in Figure 4, three sliders also generate motion straightness errors in $Y$ and $Z$ directions, namely $\delta_{Y}(x)$ and $\delta_{Z}(x)$, respectively [20,21]. Three sliders moving one after another not only cause the moving platform to produce positioning errors, but also result in straightness and orientation changes. Thus, the change of the probe position leads to measurement error. Considering the influence of $\delta_{Y}(x)$ and $\delta_{Z}(x)$, we can modify Equation (1) to:

$$
\left\{\begin{array}{l}
\left(x-x_{1}\right)^{2}+\left(y-\delta y_{1}\right)^{2}+\left(z-\delta z_{1}\right)^{2}=l_{1}{ }^{2} \\
\left(x-x_{1}\right)^{2}+\left(y+g-c-\delta y_{1}\right)^{2}+\left(z-\delta z_{1}\right)^{2}=l_{2}{ }^{2} \\
\left(x+e-x_{2}\right)^{2}+\left(y+g+f-b-c-\delta y_{2}\right)^{2}+\left(z-\delta z_{2}\right)^{2}=l_{3}{ }^{2} \\
\left(x+d+e-a-x_{2}\right)^{2}+\left(y+g+f-b-c-\delta y_{2}\right)^{2}+\left(z-\delta z_{2}\right)^{2}=l_{4}{ }^{2} \\
\left(x+d+2 e-x_{3}\right)^{2}+\left(y+g-c-\delta y_{3}\right)^{2}+\left(z-\delta z_{3}\right)^{2}=l_{5}{ }^{2} \\
\left(x+d+2 e-x_{3}\right)^{2}+\left(y-\delta y_{3}\right)^{2}+\left(z-\delta z_{3}\right)^{2}=l_{6}{ }^{2}
\end{array}\right.
$$

The analytical solution of the above set of equations is difficult to find. Only a numerical solution can be obtained by numerical iteration analysis. Other parallel mechanisms are also generally encountered. Any type of numerical algorithm in the calculation process consists of attempt, approximation, compromise, and convergence. In real parallel mechanism motion, the position of the moving platform is determined by the plurality of bars in common. In this process, mechanical tensile, compression, torsion, and elastic deformation of involved parts occur. When a certain position can achieve balance among these factors, this is the actual position that the moving platform reaches. Error averaging effect happens during this moving process. Reflecting on mathematics, it is iteration and convergence. Thus, we find that if any iterative algorithm has weak error averaging effect, it will be unsuitable for use in parallel mechanism calculation.

As mentioned by Bryan in 1979 [12], the slider motion straightness errors are different when different points on the slider are measured. We are mainly interested in the error of the point where two universal joints center on the slider. Therefore, when a dual-frequency laser straightness interferometer was used to detect slider motion straightness error, the interference mirror (Wollaston prism) required adjustment to be as close as possible to the center point of two joints on the moving slider. The large reflection mirror was statically fixed to the guide support. The experimental setup is shown in Figure 8. 


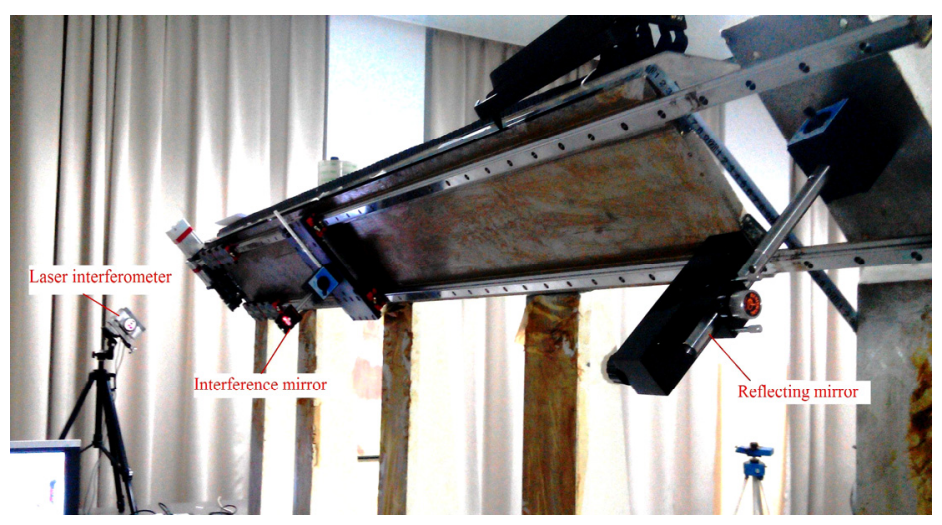

Figure 8. Straightness motion error experiment.

Straightness errors in $Y$ and $Z$ directions were captured by the interferometer (Renishaw XL-80, Renishaw, New Mills, UK). The overall trend of three slider straightness motion errors is highly similar. Figure 9 shows the straightness motion errors of first slider in two directions. The horizontal axis is the coordinate $x_{1}$ of the first slider. The interferometer picks up $\delta_{Y}(x)$ once after the slider moves $50 \mathrm{~mm}$ in one direction, thus, the "forward" curve can be established. The measurement process was repeated in the reverse direction to established the "reverse" curve. It can be seen that the two directional measurements are quite consistent, and the average value of the data is then used as the straightness motion error of the slider in the $Y$ direction, as shown in Figure 9 (above). For the straightness motion error of the slider in the $Z$ direction, we repeated the detection process, but the interference mirror and the reflection mirror needed to be rotated $90^{\circ}$ in advance. Figure 9 (below) shows the variation of $\delta_{Z}(x)$.
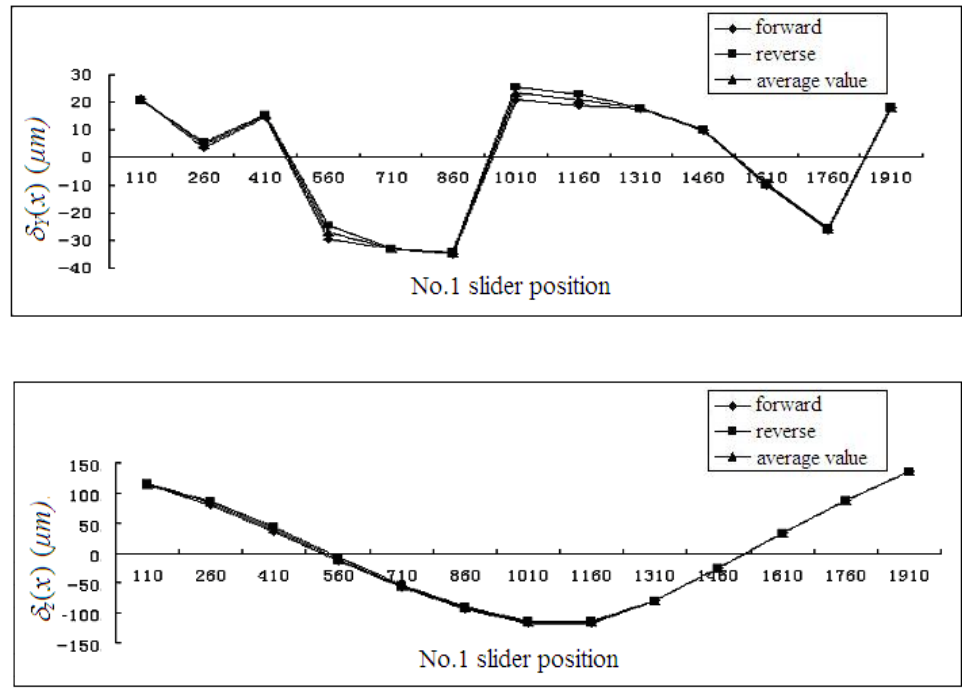

Figure 9. Straightness motion error of slider 1.

The value difference among six sets of data of straightness motion errors is extremely small. Thus, only one series of data on a slider was selected to establish the error model for the subsequent error calculation. A quadratic function was used to establish the model for straightness motion error in the $Z$ direction. The straightness motion error in the $Y$ direction is relatively complex, where the error model needs to be constructed with the third-order spline curve function.

As shown in Table 3, 3 groups $(x=750,1000,1250)$ in the measurement space were selected initially, and 9 points were selected as feature points in each group. Thus, a total of 27 points were 
distributed in the measurement space. Corresponding to each point is a slider position coordinate that can be determined through the inverse kinematics of PCMM. Putting the measured straightness errors of the three sliders into Equation (7) and solving the actual probe position in the measurement space by Equation (3), the contributions of straightness motion errors to the probe's positional error are listed in Table 3.

Table 3. Error contributed by straightness motion errors.

\begin{tabular}{|c|c|c|c|c|c|c|}
\hline \multirow{2}{*}{$\begin{array}{c}\text { Selected Points/mm } \\
(X, Y, Z)\end{array}$} & \multicolumn{3}{|c|}{ Corresponding Slider Position/mm } & \multicolumn{3}{|c|}{ Error Value/ $\mu \mathrm{m}$} \\
\hline & $x_{1}$ & $x_{2}$ & $x_{3}$ & $\delta_{x S}$ & $\delta_{y S}$ & $\delta_{z S}$ \\
\hline $750,150,200$ & 193.58 & 1167.44 & 1319.58 & 3 & 8 & -8 \\
\hline $750,150,300$ & 240.49 & 1047.23 & 1285.55 & -7 & 5 & 17 \\
\hline $750,150,400$ & 314.56 & 1074.49 & 1211.45 & 6 & 2 & -9 \\
\hline $750,300,200$ & 257.96 & 1357.98 & 1468.03 & -3 & -4 & 4 \\
\hline $750,300,300$ & 311.70 & 1308.28 & 1414.32 & 3 & -1 & 9 \\
\hline $750,300,400$ & 400.57 & 1228.27 & 1325.46 & -5 & -10 & -25 \\
\hline $750,450,200$ & 390.01 & 1371.35 & 1486.67 & -1 & 1 & 3 \\
\hline $750,450,300$ & 467.86 & 1310.48 & 1458.19 & -2 & 13 & -13 \\
\hline $750,450,400$ & 652.02 & 1203.11 & 1374.04 & 2 & -8 & 14 \\
\hline $1000,150,200$ & 443.58 & 1403.64 & 1582.45 & 4 & 8 & 6 \\
\hline $1000,150,300$ & 490.49 & 1397.26 & 1735.50 & 3 & -9 & -22 \\
\hline$\ldots$ & $\ldots$ & $\ldots$ & . . & $\ldots$ & $\ldots$ & $\ldots$ \\
\hline $1250,450,200$ & 890.00 & 1671.33 & 1836.56 & 1 & 10 & 18 \\
\hline $1250,450,300$ & 967.86 & 1610.46 & 1858.18 & 3 & -1 & 5 \\
\hline $1250,450,400$ & 1152.0 & 1653.10 & 1874.07 & 3 & 8 & -8 \\
\hline
\end{tabular}

... represents a few sets of data was omitted.

As shown in Table 3, the straightness motion errors of the sliders contribute the smallest measuring error in the $X$ direction and produce the largest error in the $Z$ direction. This means that, if the purpose is to promote the $Z$ direction measuring accuracy, the slider straightness motion error needs to be improved significantly. The error curves in three directions are shown in Figure 10. The total variation in three directions ranges from -0.03 to $\sim 0.02 \mathrm{~mm}$. Compared with the original slider motion straightness error data, the final influence of the slider motion straightness error on the measurement accuracy is relatively decreased $[9,22]$. This also demonstrates the error averaging effect for the PCMM.

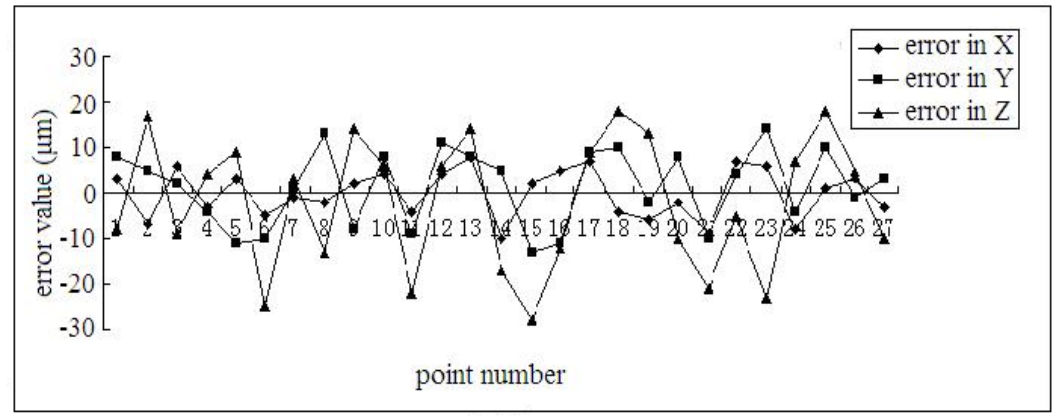

Figure 10. Variation of error caused by straightness motion error.

\section{Conclusions}

This study analyzed the error averaging effect on the parallel mechanism. Although this issue had not been fully explored, we have found solid evidence from theoretical analysis and experimental results that this effect is mainly dependent on the symmetry of structure. The input motion error, angle errors, and straightness errors of each slider can be averaged and reduced at the probe position through parallel links rods. In the 3-PUU PCMM, the precision linear guider, which is shared by 
three sliders, is an important measuring part. Our future studies on error averaging effect will further explore the correlation between different error sources and the error transfer coefficient to establish an integrated error model to improve the accuracy of the developed PCMM.

Acknowledgments: The authors are grateful for the financial support provided by the Natural Science Foundation of China $(51475133,51675157)$ and Natural Science Foundation of Anhui Province (1508085MF122).

Author Contributions: Peng-Hao Hu and Chang-Wei Yu conceived, designed the instrument structure and wrote the paper; Kuang-Chao Fan guided the instrument design and provided new ideas about the instrument structure; Xue-Ming Dang designed and produced the motion control system; Rui-Jun Li finished the experiment and processed the obtained data.

Conflicts of Interest: The authors declare no conflict of interest.

\section{Abbreviation}

The following abbreviations are used in this manuscript:

PCMM Parallel coordinate measuring machine

3-PUU 3 pairs of prismatic-universal-universal joints

\section{References}

1. Reichert, B.A.; Turney, S.M.; Chapman, D.K.; Fields, T.A. Encoder Eccentricity Correction for Motion Control Systems. U.S. Patent 2010/0072938 A1, 25 March 2010.

2. Yandayan, T.; Akgoz, S.A.; Haitjema, H. A novel technique for calibration of polygon angles with non-integer subdivision of indexing table. Precis. Eng. 2002, 26, 412-424. [CrossRef]

3. Qi, E.; Fang, Z.; Sun, T.; Chen, J. A method for predicting hydrostatic guide error averaging effects based on three-dimensional profile error. Tribol. Int. 2016, 95, 279-289. [CrossRef]

4. Fan, K.C.; Wang, H.; Zhao, J.W.; Chang, T.-H. Sensitivity analysis of the 3-PRS parallel kinematic spindle platform of a serial-parallel machine tool. Mach. Tools Manuf. 2003, 43, 1561-1569. [CrossRef]

5. Zarske, W. Device for the Translatory Position of a Platform. U.S. Patent 2004/000037663 A1, 26 February 2004.

6. Gao, F.; Li, W.; Zhao, X.; Jin, Z.; Zhao, H. New kinematic structures for 2-, 3-.4-, and 5-DOF parallel manipulator designs. Mech. Mach. Theory 2002, 37, 1395-1411. [CrossRef]

7. Hu, P.; Yang, J.; He, X. 3-PSS Parallel CMM. China Patent 201010541816, 9 May 2012.

8. $\mathrm{Hu}, \mathrm{P}$; $\mathrm{Li}, \mathrm{S}$. Kinematics solution of 3-PSS parallel mechanism and its application in parallel CMM. Opt. Precis. Eng. 2012, 20, 782-788.

9. Hu, P.; Zhang, J.; Ma, X.; Fei, Y. Analysis of Slider Motion Error on 3-PUU Parallel Coordinate Measuring Machine. J. Mech. Eng. 2015, 51, 45-50. [CrossRef]

10. Liu, X.; Wang, J.; Li, J.; Gao, F. On the Workspace of a Novel Spatial 3-DOF Parallel Manipulator. J. Mech. Eng. 2012, 37, 36-39. [CrossRef]

11. Li, S.; Hu, P. Structure Optimization of Parallel CMM. Opt. Precis. Eng. 2013, 21, 138-145.

12. Bryan, J.B. The Abbe Principle Revised-An updated Interpretation. Precis. Eng. 1979, 3, 129-132. [CrossRef]

13. Gao, W.; Lee, J.C.; Araia, Y.; Noha, Y.J.; Hwangb, J.H.; Parkb, C.H. Measurement of Slide Error of an Ultra-precision Diamond Turning Machine by Using a Rotating Cylinder Workpiece. Int. J. Mach. Tools Manuf. 2010, 50, 404-410. [CrossRef]

14. Gao, W.; Araia, Y.; Shibuya, A.; Kiyonoa, S.; Parkb, C.H. Measurement of Multi-degree-of-freedom Error Motions of a Precision Linear Air-bearing Stage. Precis. Eng. 2006, 30, 96-103. [CrossRef]

15. Fan, K.; Chen, M.-J. A 6-degree-of-freedom Measurement System for the Accuracy of X-Y Stages. Precis. Eng. 2000, 24, 15-23. [CrossRef]

16. Kim, J.-A.; Kima, J.-A.; Baea, E.W.; Kima, S.H.; Kwak, Y.K. Design Methods for Six-degree-of-freedom displacement Measurement System Using Cooperative Targets. Precis. Eng. 2002, 26, 99-104. [CrossRef]

17. Zhang, G. A Study on the Abbe Principle and Abbe Error. CIRP Ann. 1989, 38, 525-528. [CrossRef]

18. Ekinci, T.O.; Mayer, J.R.R. Relationships between straightness and angular kinematic errors in machines. Int. J. Mach. Tools Manuf. 2007, 47, 1997-2004. [CrossRef]

19. Li, S.; Li, S.; Zhao, P.; Hu, P. Key Errors Analysis and Simulation of a 3-PUU Parallel CMM. In Proceedings of the 6th International Symposium on Precision Mechanical Measurements, Guiyang, China, 8 August 2013. 
20. Hu, P.; Yao, L.; Li, S. Research of a Novel CMM with 3-PSS Parallel Mechanism. In Proceedings of the 7th International Symposium on Precision Engineering Measurements and Instrumentation, Lijiang, China, 7-11 August 2011.

21. Zhang, J.; Hu, P.; Ma, X. 3-PUU Abbe error analysis on 3-PUU parallel CMM. China Sci. Pap. 2015, 10, 471-474.

22. Majda, P. Modeling of geometric errors of linear guideway and their influence on joint kinematic error in machine tools. Precis. Eng. 2012, 36, 369-378. [CrossRef] 